\title{
EDITORIAL
}

\section{Profiling circulating tumour cells and cell free DNA together in metastatic colon cancer}

The characterisation of CTCs (circulating tumour cells) and cfDNA (circulating free DNA) by Salvianti et al. highlight critical aspects of these approaches' relative strengths, weaknesses, and interdependencies in this study.

British Journal of Cancer (2021) 125:907-908; https://doi.org/10.1038/s41416-021-01397-8

\section{MAIN}

In the study by Salvianti et al. ${ }^{1}$ appearing in the latest issue of the British Journal of Cancer, 20 patients at the Medical Oncology Unit of AOUC of Florence, Italy, with NRAS or KRAS mutated metastatic colorectal cancer (mCRC) were enrolled. CTC enumeration was successful in $90 \%$ of the cohort (18 out of 20 patients) using the CellSearch system but only two of these had, as far as we can tell, enough CTCs for single cell analysis. As per other studies, Kaplan-Meier estimates of OS (overall survival) and PFS (progression-free survival) confirmed the authors' view that CTC enumeration could be deployed as a standalone prognostic indicator. Similarly, sequencing of cfDNA provided sufficient coverage to deduce a panel of genes of interest in $95 \%$ (19 out of 20 patients) and cfDNA levels were also shown to be a prognostic marker in patients with $\mathrm{mCRC}$. Both appear to be reliable and reproducible markers in this cohort, in those individuals with a positive reading.

Further studies with larger sample sizes require exploration. Interestingly here, some of the samples shared the SMAD4 R361H mutation frequently detected in CRC, associated with a decline in OS $(P=0.02){ }^{1}$ Other studies have indicated that this specific mutation in exon 8 occurs in later stage $m C R C$, increasing with disease progression, a finding which again is complicated by small cohorts. ${ }^{2}$ The SMAD4 R361H mutation may serve as a useful marker in disease monitoring via the sort of liquid biopsy workflow identified in this research. ${ }^{2}$ Given that responses to second-line chemotherapy in $\mathrm{MCRC}$ patients tends to be poorer compared with first-line chemotherapy, the SMAD4 R361H might serve as a marker to monitor clinical responses, an improvement to the current practice of observation of tumour shrinkage as the main 'efficacy endpoint', tumour markers and quality of life parameters notwithstanding.

Contrary to expected findings, the $40 \%$ frequency of mutations in the tumour suppressor gene FBXW7 was greater than previously reported (10\%). Intriguingly, the variant (p.465c) was identified in $>75 \%$ of the FBXW7 mutants (seven of nine samples) in the cohort. Previous research indicates that the FBXW7-NOTCH-CCL2 pathway plays a role in the regulation of metastasis. ${ }^{3,4}$ The identification of p.465c as a subtype of interest in this research will facilitate further work to determine if FBXW7 is implicated in drug resistance in this setting. ${ }^{3,4}$

Concordance between variants found in FFPE (formalin fixed paraffin embedded) tissue samples and cfDNA measured $84 \%$ (16 out of 19 samples). Liquid biopsy also identified TP53 (70\%), APC (50\%), FBXW7 (10\%), PIK3CA (10-15\%), and SMAD4 (10\%), as common sequence variants. Evaluation of sequence variants in cfDNA in combination with analyses of NRAS and KRAS identified in tissue samples as part of a larger cohort would confirm the role of these mutants as independent indicators of prognosis in $\mathrm{mCRC}$ patients. A larger sample size would also present the opportunity to analyse a greater number of CTCs to further our understanding of the relationship between cfDNA and CTCs.

Longitudinal monitoring of two patients using CTC and CfDNA, revealed markers that might predict successful treatment with VEGF inhibitors. The first case, a 53-year-old female, received 12 cycles of first-line FOLFOXIRI/bevacizumab followed by 5-FU/ bevacizumab maintenance obtaining a partial response. Simultaneously, both CTCs and KRAS (p.G12S) mutations in cfDNA were undetectable in the patient at 40 days. After 6 months of treatment, APC and KRAS mutations in cfDNA increased prior to progressive disease (PD) detection via scanning. The second case, a 60-year-old male, positive for KRAS G13D with no APC mutation, demonstrated PD after six cycles of first-line FOLFOX bevacizumab. From an observational perspective, KRAS and APC mutations may be markers for disease that is more readily treatable with VEGF inhibitors.

The liquid biopsy story is entering the clinical domain in real time, no pun intended. Droplet digital PCR (ddPCR) assays have been suggested as a tool to improve the sensitivity of cfDNA analysis. ${ }^{5}$ For example, a KRAS exon 2 mutation with a variant allele frequency $\geq 0.1 \%$ was identified in $90 \%$ of patients at baseline by Bidard et al. in the PRODIGY-14 trial. ${ }^{5,6}$ Taken in combination with advances in next-generation liquid biopsies, labon-a-chip diagnostics allowing for the amplification of cfDNA, it seems likely that sequencing methods with increased sensitivity to overcome these challenges, are on the horizon. ${ }^{7}$ An emerging protocol of sequencing concatenated copies of DNA relative to a consensus sequence derived via consensus calling provides a solution, at least in part. Scalable, inexpensive technology enabled solutions such as Oxford Nanopore's MinION, capable of detecting small fragments of DNA albeit via long reads and amplified loci of interest could potentially allow for targeted rapid sequencing at both high specificity and sensitivity. The benefit of enhanced cfDNA analysis does not remove the requirement for CTC evaluation; this is evidenced via CTC data highlighting the mutational heterogeneity of single EpCAM-positive CTCs, albeit again in a limited number of samples. ${ }^{4,6}$ Moreover, findings here indicate that variability of driver gene mutation rates seen in cfDNA derive from the different pre-analytical steps taken and could be improved through methodology refinement as highlighted by the authors in the discussion. Variation in protocols is 
invariably a feature of liquid biopsy studies, despite standard operating procedures being shared.

The data presented in this study ${ }^{1}$ confirm that CTC changes during and following chemotherapy treatment are associated with PFS and OS. Additionally, the authors demonstrate that cfDNA analysis in combination with CTC evaluation might improve prognostic accuracy, though the future focus of liquid biopsies in general will be on prediction and diagnosis. Given these findings, it is likely that stratification of $\mathrm{mCRC}$ patients would be possible in a larger cohort of patients as has been achieved with other cancer types, using cfDNA and CTCs together. ${ }^{8-11}$ Molecular characterisation of CTCs and cfDNA in a larger cohort would indeed confirm analytical and clinical validity of the observations in this study; our strong preference is such data that are longitudinal with at least one further time point.

\section{AUTHOR CONTRIBUTIONS}

The authors contributed equally to this work and wrote the manuscript together and approved the final version.

\section{ADDITIONAL INFORMATION}

Ethics approval and consent to participate Not relevant.

Consent to publish Not applicable.

Data availability Not applicable.

Competing interests J. Stebbing's conflicts can be found at www.nature.com/onc/ editors, but none are relevant to this piece. T. Friend is now an employee of Oxford Nanopore Technologies Ltd. but was not engaged with the company at the time of writing.

Funding information The authors received no funding for this work.

Publisher's note Springer Nature remains neutral with regard to jurisdictional claims in published maps and institutional affiliations.
Thomas Friend (D) and Justin Stebbing (D)

${ }^{1}$ Department of Surgery and Cancer, Imperial College, London, UK Correspondence: Thomas Friend (tcf2111@columbia.edu)

\section{REFERENCES}

1. Salvianti, F., Gelmini, S., Mancini, I., Pazzagli, M., Pillozzi, S., Giommoni, E. et al. Circulating tumour cells and cell-free DNA as a prognostic factor in metastatic colorectal cancer: the OMITERC prospective study. Br. J Cancer. https://doi.org/ 10.1038/s41416-021-01399-6. (2021) in the press

2. Bai, J., Gao, J. \& Mao, Z. Genetic mutations in human rectal cancers detected by targeted sequencing. J. Hum. Genet. 60, 589-596 (2015).

3. Wu, Y., Chang, N., Zhang, Y., Zhang, X., Xu, L., Che, Y. et al. METTL3-mediated m6A mRNA modification of FBXW7 suppresses lung adenocarcinoma. J. Exp. Clin. Cancer Res. 40, 90 (2021).

4. Jardim, D. L., Wheler, J. J., Hess, K., Tsimberidou, A. M., Zinner, R., Janku, F. et al. FBXW7 mutations in patients with advanced cancers: clinical and molecular characteristics and outcomes with mTOR inhibitors. PLoS ONE 9, e89388 (2014).

5. Ignatiadis, M., Sledge, G. W. \& Jeffrey, S.S. Liquid biopsy enters the clinicimplementation issues and future challenges. Nat. Rev. Clin. Oncol. PMID: 33473219. https://doi.org/10.1038/s41571-020-00457-x (2021).

6. Bidard, F. C., Kiavue, N., Ychou, M., Cabel, L., Stern, M.-H., Madic. J. et al. Circulating tumor cells and circulating tumor DNA detection in potentially resectable metastatic colorectal cancer: a prospective ancillary study to the Unicancer PRODIGY-14 trial. Cells 8, 516 (2019).

7. Shaw, J., Guttery, D., Hills, A., Fernandez-Garcia, D., Page, K., Rosales, B. M. et al. Mutation analysis of cell-free DNA and single circulating tumor cells in metastatic breast cancer patients with high circulating tumor cell counts. Clin. Cancer Res. 23, 88-96 (2017).

8. Hodgkinson, C. L., Morrow, C. J., Li, Y., Metcalf, R. L., Rothwell, D. G., Trapani, F. et al. Tumorigenicity and genetic profiling of circulating tumor cells in small-cell lung cancer. Nat. Med. 20, 897-903 (2014).

9. Polzer, B., Medoro, G., Pasch, S., Fontana, F., Zorzino, L., Pestka, A. et al. Molecular profiling of single circulating tumor cells with diagnostic intention. EMBO Mol. Med. 6, 1371-1386 (2014).

10. Coombes, R., Page, K., Salari, R., Hastings, R. K., Armstrong, A., Ahmed, S. et al. Personalized detection of circulating tumor DNA antedates breast cancer metastatic recurrence. Clin. Cancer Res. 25, 14 (2019).

11. Bidard, F., Peeters, D., Fehm, T., Nolé, F., Gisbert-Criado, R., Mavroudis, D. et al. Clinical validity of circulating tumour cells in patients with metastatic breast cancer: a pooled analysis of individual patient data. Lancet Oncol. 15, 406-414 (2014). 